\title{
A Self-Improving Convolution Neural Network for the Classification of Hyperspectral Data
}

\author{
Pedram Ghamisi, Member, IEEE, Yushi Chen, Member, IEEE, Xiao Xiang Zhu, Senior Member, IEEE
}

\begin{abstract}
In this paper, a self-improving convolutional neural network (CNN)-based method is proposed for the classification of hyperspectral data. This approach solves the so-called curse of dimensionality and the lack of available training samples by iteratively selecting the most informative bands suitable for the designed network via fractional order Darwinian particle swarm optimization (FODPSO). The selected bands, then, are fed to the classification system to produce the final classification map. Experimental results have been conducted with two well-known hyperspectral data sets; Indian Pines and Pavia University. Results indicate that the proposed approach significantly improves a CNN-based classification method in terms of classification accuracy. In addition, this paper uses the concept of dither for the first time in the remote sensing community to tackle overfitting.
\end{abstract}

Index Terms-Hyperspectram image classification, Deep Learning, CNN, Feature Selection, FODPSO.

\section{INTRODUCTION}

$\mathbf{C}$ OMPLEX light scattering mechanisms in natural objects (e.g., vegetation), different atmospheric scattering conditions, and intra-class variability make the hyperspectral imaging process inherently nonlinear. It is believed that deep architectures can lead to progressively more abstract features at higher layers of features, and more abstract features are generally invariant to most local changes of the input.

Deep learning is characterized by the so-called "deep" neural network (DNN) architectures, i.e., deeper than three layers. If designed and trained properly, a DNN provides a hierarchical description of the input data in terms of relevant and easy to interpret features at every layers.

Depending on the architecture and the involved activation functions, several classes of DNNs have been developed such as: deep belief networks (DBN) [1], deep Boltzmann machines (DBM) [2], and autoencoders (AE) [3]. The use of deep learning for hyperspectral image analysis is still in its beginning period and the number of research works in this field is very limited. In [4], a stacked AE-based approach was introduced for hyperspectral data classification. In [5], a DBNbased feature extraction was proposed for the classification of hyperspectral data. In those approaches, however, there is a full connection between different layers and consequently, they

Pedram Ghamisi and Xiao Xiang Zhu are with German Aerospace Center (DLR), Remote Sensing Technology Institute (IMF) and Technische Universität München (TUM), Signal Processing in Earth Observation, Munich, Germany (corresponding authors, e-mail: pedram.ghamisi@dlr.de and xiao.zhu@dlr.de).

Yushi Chen is with the Department of Information Engineering, Harbin Institute of Technology. (e-mail: chenyushi@hit.edu.cn).

This research has been partly supported by Alexander von Humboldt Fellowship for postdoctoral researchers, and Helmholtz Young Investigators Group "SiPEO" (VH-NG-1018, www.sipeo.bgu.tum.de). demand to train a lot of parameters, which is an undesirable factor due to the lack of available training samples.

Among deep approaches, CNNs have attracted many researchers since they use local connections to handle spatial dependencies. In addition, CNNs share weights which significantly deceases the number of parameters needed to be trained in comparison with other deep approaches. However, the number of parameters is still high when one needs to deal with hyperspectral data. Inappropriate weights may cause getting trapped in a local minimal of the loss function. To obtain proper weights, a lot of training samples are needed for the training procedure. However, the number of available training samples is usually limited, which downgrades the result of most supervised learning approaches. Recently, few regularization methods have been introduced in order to deal with overfitting problems including L2 regularization and dropout [6]. Due to the high spectral dimensionality and the limited training samples in hyperspectral data, however, the existing regularization methods cannot handle the overfitting problem properly.

To address the issue of imbalance between dimensionality and the number of available training samples, different feature selection approaches can be considered. Conventional feature selection techniques usually demand many samples in order to accurately estimate statistics [7]. Moreover, most of those approaches are based on exhaustive search to find the best set of features among the whole dimensionality, which requires a huge CPU processing time and many RAMs in order to successfully lead to a conclusion [7]. To address the aforementioned issues, the new trend for feature selection is based on evolutionary-based optimization approaches, such as genetic algorithm (GA) and Particle Swarm Optimization (PSO) [8]. For example in [9], a GA was used to regulate hyperplane parameters of an SVM, while it finds efficient features to be fed to the classifier.

PSO has a simple concept, which can be implemented in a few lines of code. Moreover, PSO has a memory of past iterations. However, the main disadvantage of PSO is the premature convergence of the swarm. The main reasons of this disadvantage are: (i) particles try to converge to a single point, which is located on a line between the global best and the personal best positions. This point, however, is not guaranteed to be a local optimum [7], and (ii) the fast rate of information flow between particles leads to the creation of similar particles, which results in a loss in diversity. In [10], an algorithm was proposed for feature selection based on fractional order Darwinian particle swarm optimization (FODPSO) to address the main shortcoming of the PSO, i.e., 
the premature convergence of a swarm.

This paper proposes a classification approach, here entitled as self-improving CNN (SICNN), which is based on considering FODPSO as feature selection, and the classification accuracy of $\mathrm{CNN}$ on validation samples as fitness value. The proposed approach addresses the main shortcoming of a $\mathrm{CNN}$ based classification approach for hyperspectral data, i.e., the lack of available training samples, by automatically selecting the best set of bands from the whole dimensionality suitable for the defined network. The proposed approach is applied to Indian Pines and Pavia University using the standard training and test samples. It should be noted that, in this paper, feature selection has been used for the first time to improve the capability of deep networks in the hyperspectral community. In addition, this paper considers the concept of dither [11] as a regularization method for the first time in the remote sensing community to further address the issue of overfitting for CNN.

The rest of the paper is organized as follows: Section II introduces the methodology of the paper. Section III is devoted to experimental results. The main concluding remarks are mentioned in Section IV.

\section{Methodology}

We believe that there are two possible ways to cope with deep approaches for the classification of hyperspectral data: (1) one can experimentally modify the architecture of the network and set the parameters in a trial and error way, which is, of course, time demanding. It is easy to understand that the network defined in this way needs to be changed from one data to another and from one set of training samples to another one due to the lack of generalization. (2) One can define a fix yet logical network and define the most suitable bands based on the network. This paper follows the second possible way.

Fig. 1 depicts the main work flow of the proposed approach. In this paper, the performance of the $\mathrm{CNN}$ is improved via an FODPSO-based feature selection. In more detail, the overall accuracy (OA) of CNN on validation samples is improved toward the optimization process by iteratively selecting the most informative features in terms of the OA. Finally, a CNN is applied to the selected bands and the obtained result is evaluated on the test samples. In the following sections, FODPSObased feature selection and $\mathrm{CNN}$-based classification, which are the backbones of SICNN, will be elaborated on.

CNNs have a unique architecture in comparison with other deep models by using local connections and shared weights. In $\mathrm{CNN}$, some connections between neurons are replicated across the entire layer, which share the same weights and biases. ${ }^{1}$ A deep $\mathrm{CNN}$ is composed of several convolutional and pooling layers to form a deep architecture followed by a fully connected logistic regression (LR) layer at the end. We first define the convolutional layer as follows:

$$
x_{j}^{l}=f\left(\sum_{i=1}^{M} x_{i}^{l-1} * k_{i j}^{l}+b_{j}^{l}\right),
$$

${ }^{1}$ It should be noted that the concept of end-to-end training has been recently introduced to obtain deeper networks and have fewer parameters to be trained to further improve the ability of CNNs.

\section{Self-Improving CNN}

(1) Split the training set to two sets: training and validation

(2) Run FODPSO with CNN+LR as the corresponding fitness criterion as follows until the maximum number of iterations is met.

\begin{tabular}{lc|}
\hline Main program loop & Evolve swarm algorithm \\
\hline For each swarm in the collection & For each particle in the swarm \\
Evolve the swarm (Evolve & Update Particles' Fitness using the OA of \\
Swarm Algorithm: right) & the CNN+LR on validation samples \\
Allow the swarm to spawn & Update Particles' Best \\
Delete "failed" swarms & Move Particle \\
& If swarm gets better \\
& Reward swarm: spawn particle: \\
& extend swarm life \\
& If swarm has not improved \\
& Punish swarm: possibly delete \\
& particle: reduce swarm life \\
\hline
\end{tabular}

(3) Choose the best particle with the highest fitness as the output (4) Use the whole training set to train $\mathrm{CNN}+\mathrm{LR}$ on the selected bands from (3).

(5) Evaluate the final classification results using test samples.

Fig. 1. The general idea of the SICNN.

where $x_{i}^{l-1}$ represents the $i$-th feature map of (l-1)-th layer. $x_{j}^{l}$ is the $j$-th feature map of the current $(l)$-th layer, and $M$ denotes the number of input feature maps. $k_{i j}^{l}$ and $b_{j}^{l}$ are the trainable parameters in the convolutional layer. $f($.$) is a$ nonlinear function and $*$ is the convolution operation.

Pooling can extract invariant features by reducing the resolution of feature maps. Each pooling layer corresponds to the previous convolutional layer, while it combines a small $N \times 1$ patch of the convolution layer. The most common pooling technique is max pooling.

To handle the issue of overfitting to some extent, we have considered dropout, and to accelerate the convergence, a rectified linear unit (ReLU) is used. There are different types of ReLUs to be considered in the network. In this study, a simple nonlinear ReLU operation was considered, which accepts the input of a neuron if it is positive, while it returns 0 if the input is negative. Dropout sets the output of some hidden neurons to zero, and the dropped neurons do not contribute in the forward pass as well as the back propagation procedure. At different training epochs, the deep CNN introduces different neural networks by dropping neurons in a random way. By using ReLU and dropout, the outputs of many neurons turn to 0 . The consideration of the ReLU and dropout at several layers build up a powerful sparse-based regularization for the deep network and address the overfitting problem for hyperspectral image classification.

In nonlinear signal processing, the use of additive noise prior to nonlinear processing can decorrelate nonlinear distortion products. Such process is regarded as dithering. In this context, DNNs can be considered as discrete (sampled) systems composing of linear filters and nonlinear demodulation stages. As mentioned in [12], the so-called inherent nonlinear distortion and aliasing lead to the issue of overfitting. Therefore, if dither can suppress nonlinear distortion and aliasing, it might also be a useful tool to regularize the CNN. As a result, to further mitigate the issue of overfitting, in 
addition to dropout, we introduce a simple yet effective method named dither, based on [11], to improve the regularization procedure. New sample $\mathbf{y}_{m}$ is obtained by adding random noise to a training sample $\mathbf{x}_{m}$ as $\mathbf{y}_{m}=\mathbf{x}_{m}+\beta \mathbf{n}$, in which $\mathbf{n}$ is the additive noise, while $\beta$ controls the intensity of the random Gaussian noise n.

In this paper, the output of $\mathrm{CNN}$ is classified using an LR, which employs soft-max as its output-layer activation. Soft-max ensures the activation of each output unit sums to one. Therefore, the output can be seen as a set of conditional probabilities. Given the input vector $R$, the probability that the input sample belongs to class $i$ is estimated as follows:

$$
P(Y=i \mid R, W, b)=s(W R+b)=\frac{e^{W_{i} R+b_{i}}}{\sum_{j} e^{W_{j} R+b_{j}}}
$$

where $W$ and $b$ are weights and biases of the LR layer, respectively, while the summation is done over all the output units. LR can be considered as a single layer neural network and as a result, it can be merged with the $\mathrm{CNN}$ to form a CNN+LR deep classifier. In this manner, the size of the outputlayer should be equal to the number of classes.

\section{A. FODPSO-based Feature Selection}

FODPSO was proposed in [13] for image segmentation. FODPSO can overcome the main drawback of a simple PSO, i.e., the stagnation of particles around sub-optimal solutions by considering two main modifications:

1) FODPSO run many simultaneous parallel PSO algorithms, each one as a different swarm, on the same test problem and then a simple natural selection mechanism is applied. When a search tends to a sub-optimal solution, the search in that area is simply discarded and another area is searched instead. In this approach, at each step, swarms that get better are rewarded (extend particle life or spawn a new descendent) and swarms that stagnate are punished (reduce swarm life or delete particles). For more information, please see [13].

2) Fractional calculus is used to control the convergence rate of the algorithm. This method has been further investigated for image segmentation and feature selection in $[10,13]$. The main advantage of fractional calculus is that while an integer-order derivative just implies a finite series, the fractional-order derivative requires an infinite number of terms. More precisely, integer derivatives are "local" operators, while fractional derivatives have, implicitly, a "memory" of all past events, which is useful to control the dynamic of each swarm.

For detailed information about the FODPSO-based feature selection please see [10]. In order to consider the concept of the FODPSO for feature selection, the following points need to be considered:

1) The dimension of each particle should be equal to the number of bands. Therefore, the velocity dimension ( $\mathrm{dim}$ $\left.v_{n}[t]\right)$, as well as the position dimension ( $\left.\operatorname{dim} \mathbf{x}_{n}[t]\right)$, at iteration $t$, are equal to the total number of bands of the hyperspectral image, i.e., $\operatorname{dim} v_{n}[t]=\operatorname{dim} \mathbf{x}_{n}[t]=l$.
2) Since FODPSO is used here for band selection, each particle should be represented its position in binary values, in which 0 shows the absence of the corresponding band, while 1 shows the presence of the corresponding band. In this case, as mentioned in [10], the velocity of a particle can be considered as a probability to change the binary value from 0 to 1 or vice versa. In order to obtain a binary position, one can, first, normalize velocities in the range of 0 and 1 as follows:

$$
\Delta \mathbf{x}_{n}[t+1]=\frac{1}{1+e^{-v_{n}[t+1]}}
$$

Consequently, the position of each particle can be represented as follows:

$$
\mathbf{x}_{n}[t+1]= \begin{cases}1, & \Delta \mathbf{x}_{n}[t+1] \geq r \\ 0, & \Delta \mathbf{x}_{n}[t+1]<r\end{cases}
$$

wherein $r$ is a random $l$-dimension vector with each component generally a uniform random number between 0 and 1 .

3) The OA of CNN+LR on validation samples can be considered as the fitness value.

\section{EXPERIMENTAL RESULTS}

In this paper, two widely used hyperspectral data have been used. The first data set (Indian Pines) was captured by AVIRIS over a rural area in NW Indiana. For this data set, 200 data channels are used after the removal of the spectral bands affected by atmospheric absorption. The second data set (Pavia University) is of the Engineering School at the University of Pavia captured by ROSIS-03. In the experiments, 12 noisy data channels are eliminated, and 103 data channels are used for processing. We intentionally selected these two data sets since they are different in many aspects such as spatial resolution, number of bands and the type of the scene and land-covers, which can be useful to evaluate the generalization capability of the proposed method.

In order to evaluate the capability of SICNN in an ill-posed situation (i.e., when there is no balance between the number of training samples and bands), and make the approach fully comparable with the-state-of-the-arts, for both data sets, the standard sets of training and test samples have been taken into account. For detailed information about the data sets and their corresponding test and training samples, please see [14]. The total number of training and test samples for Indian Pines are 695 and 9671, respectively, while for Pavia University are 3912 and 42776, respectively.

Deep learning uses deep neural networks, which are inherently parallel algorithms. To accelerate the training time, "matconvnet-1.0-beta18" with CUDA configuration is adapted.

In order to keep the borders of different features through convolutional layers, the original image is padded with an extra artificial border, which mirrors the original border.

In this work, $90 \%$ of the training samples have been used to train weights and biases and the rest as validation samples to guide the design of a proper architecture in order to avoid overfiting. It should be noted that the validation samples are 
different from the test samples. The validation samples have been extracted directly from the training set.

In this paper, we only included the classification accuracy of the SVM and RF (as two strong classifiers to handle high dimensional data with a limited number of training samples [7]) to show that the proposed method is also capable of handling ill-posed situations, while a direct comparison of these approaches with SICNN is not expected. For the SVM, an RBF kernel is taken into account and the hyperplane parameters have been adjusted via five-fold cross validation. The number of trees for RF is set to 200. For the FODPSO, we used the same set of parameters as [10].

The input hyperspectral data sets were normalized in the range of $\left[\begin{array}{ll}-0.5 & 0.5\end{array}\right]$. To exploit sufficient spatial information for the pixel to be classified, a large patch with the size of $27 \times 27$ was used. Since the studied areas are of small sizes, only three convolution layers and two pooling layers have been used. Details of the architecture for the CNN are listed in Fig. 2. It should be noted that the same network has been used for both data sets.

In the training procedure, a mini batch with a size of 32 was used. For relatively small training samples, as in our case, this could allow the training step to perform more frequent parameter updates and achieve faster convergence in practice. In addition, a dynamic learning rate is adopted in our case. The whole training period is divided into five stages, starting from a learning rate of 0.01 , and decreasing by half for the subsequent stage. This setting provides a fast descend of loss function at the beginning, while the gradual decreasing rate can ensure a small but consistent progress. After reaching a certain stage of training, a big rate may no longer be suitable since it causes oversteps and gives a higher loss. This way of adjusting the learning rate enables the algorithm to be converged in a very few iterations. For Indian Pines, the inner number of iterations (CNN as the fitness metric for feature selection) is set to 20 while the outer number of iteration (for the final classification of the selected bands) is set to 80. For Pavia University, the inner and outer numbers of iterations are set to 10 and 20, respectively. The difference between the inner and outer number of iterations is due to the fact that the number of training samples for the inner feature selection is significantly fewer than the ones for the outer classification step. Hence, the number of demanded iterations is also lower. The reason why the number of iterations is different for Indian Pines and Pavia University lies on the differences between the data sets, i.e., the number of bands and classes.

In this paper, RF, SVM, and $\mathbf{C N N + L R}$ refer to situations where the input data sets are classified by RF, SVM, and CNN+LR respectively. SICNN dither $_{\text {and }}$ SICNN represent the performance of the proposed approach, with and without dither, respectively.

\section{A. Experimental Results}

The obtained classification accuracies for both data sets have been reported in Table I. The results show that the proposed SICNN can improve the classification accuracy of $\mathbf{C N N + L R}$, $\mathbf{R F}$, and SVM in almost all situations. The only exception is

\begin{tabular}{|c|c|}
\hline INPUT & {$[27 \times 27 \times 10]$} \\
\hline CONV-1 & kernel size: $4 \times 4 \times 10$ kernel \#: 32 weights: $(4 \times 4 \times 10) \times 32+32$ (bias) \\
\hline Feature Map-1 & {$[24 \times 24 \times 32]$} \\
\hline POOL-1 & size: $2 \times 2$ \\
\hline Feature Map-2 & {$[12 \times 12 \times 32]$} \\
\hline CONV-2 & kernel size: $5 \times 5 \times 32$ kernel \#: 64 weights: $(5 \times 5 \times 32) \times 64+64$ (bias) \\
\hline Feature Map-3 & {$[8 \times 8 \times 64]$} \\
\hline POOL-2 & size: $2 \times 2$ \\
\hline Feature. Map-4 & {$[4 \times 4 \times 64]$} \\
\hline CONV-3 & kernel size: $4 \times 4 \times 64$ kernel \#: 128 weights: $(4 \times 4 \times 64) \times 128+128$ (bias) \\
\hline Feature Map-5 & {$[1 \times 1 \times 128]$} \\
\hline Full Connection & kernel size: $1 \times 1 \times 128$ kernel \#: 9 weights: $(1 \times 1 \times 128) \times 16+16$ (bias) \\
\hline Feature Map-6 & {$[1 \times 1 \times 16]$} \\
\hline \multicolumn{2}{|l|}{ Softmaxloss } \\
\hline Output & probability vector: $[1 \times 16]$ \\
\hline
\end{tabular}

Fig. 2. Detailed information about the network considered for CNN and SICNN.

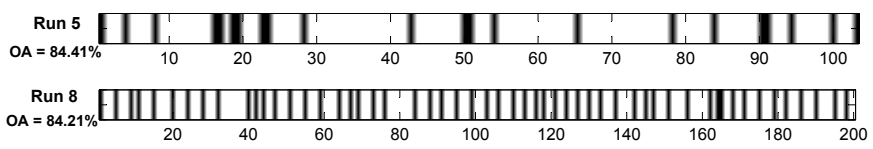

Fig. 3. Selected bands by the proposed approach using dither; from top to bottom: the fifth run on Pavia University and the eight run on Indian Pines.

that SVM yields the best AA compared to other approaches for Pavia University.

According to Table I, in most cases, the SVM can outperform the CNN+LR. The reason is that for SVMs, in order to train the classifier, only samples that are close to the class boundary (support vectors) are needed to locate the hyperplane vector in the feature space. Therefore, SVMs can efficiently handle high dimensional data with a limited number of training samples. However, the CNN needs to train a huge number of parameters and consequently, many training samples are demanded. With reference to Table I, this problem can be addressed to a great extent using the proposed approach combined by the FODPSO. In more detail, the FODPSO can iteratively selects the most appropriate set of bands suitable for the network.

Table II gives information about classification accuracies obtained by CNN+LR and SICNN with the dither. With reference to Tables I and II, one can notice an improvement in terms of classification accuracy using this regularization approach. The main reason of this improvement is that dither helps the CNN+LR and SICNN to tackle overfitting and be converged quickly. As mentioned, the inner number of iterations for Indian Pines and Pavia are set to 20 and 10, respectively. However, this numbers might not be enough to guarantee the convergence of each swarm appropriately. Dither leads to a fast learning and convergence, which is beneficial for the proposed approach. Fig. 3 demonstrates the sets of bands with the highest OA among 10 runs selected by the proposed approach SICNN $\mathbf{N}_{\text {dither. }}$. The OA of each set is also shown in the figure. The number of selected bands for Pavia University and Indian Pines are 22 and 55, respectively.

\section{CONCLUSION}

In this paper, a self-improving hyperspectral classification approach is proposed, which is based on a deep CNN and 
TABLE I

CLASSIFICATION ACCURACIES OF DIFFERENT APPROACHES INCLUDING: RF, SVM, CNN+LR AND SICNN FOR BOTH INDIAN PINES AND PAVIA UNIVERSITY. THE BEST RESULT IS SHOWN IN BOLD TYPE FACE. FOR CNN+LR AND SICNN, THE AVERAGE VALUES OF 10 RUNS ARE REPORTED AND THE STANDARD DEVIATION OF THE RUNS HAVE BEEN SHOWN IN BRACKETS.

\begin{tabular}{|c|c|c|c|c|c|c|c|c|}
\hline & \multicolumn{3}{|c|}{ Indian Pines } & \multicolumn{3}{c|}{ Pavia University } \\
\hline Metric & SVM & RF & CNN+LR & SICNN & SVM & RF & CNN+LR & SICNN \\
\hline Overall Accuracy(\%) & 78.20 & 70.24 & $74.44(0.81)$ & $\mathbf{8 1 . 6 6 ( 1 . 7 0 )}$ & 78.21 & 71.64 & $78.45(1.04)$ & $\mathbf{8 2 . 6 7}(\mathbf{1 . 2 7})$ \\
\hline Average Accuracy(\%) & 86.00 & 76.98 & $85.39(0.58)$ & $\mathbf{8 9 . 6 4 ( 1 . 1 1 )}$ & $\mathbf{8 7 . 1 4}$ & 82.25 & $79.05(0.67)$ & $82.18(0.59)$ \\
\hline Kappa Coefficient & 0.75 & 0.6642 & $0.7123(0.0093)$ & $\mathbf{0 . 7 9 3 3}(\mathbf{0 . 0 1 6 5})$ & 0.7333 & 0.6511 & $0.7312(0.0093)$ & $\mathbf{0 . 7 7 1 6}(\mathbf{0 . 0 1 5 6})$ \\
\hline
\end{tabular}

TABLE II

THE SIGNIFICANCE OF USING DITHER ON CLASSIFICATION ACCRACIES.

\begin{tabular}{|c|c|c|c|c|}
\hline & \multicolumn{2}{|c|}{ Indian Pines } & \multicolumn{2}{c|}{ Pavia University } \\
\hline Metric & CNN+LR $_{\text {dither }}$ & SICNN $_{\text {dither }}$ & CNN+LR $_{\text {dither }}$ & SICNN $_{\text {dither }}$ \\
\hline Overall Accuracy(\%) & $75.27(0.56)$ & $83.26(1.19)$ & $78.81(0.89)$ & $83.41(0.79)$ \\
\hline Average Accuracy(\%) & $86.11(0.64)$ & $91.07(0.85)$ & $79.73(0.91)$ & $83.04(0.82)$ \\
\hline Kappa Coefficient & $0.7181(0.0143)$ & $0.8092(0.0083)$ & $0.7342(0.0142)$ & $0.7784(0.0091)$ \\
\hline
\end{tabular}

FODPSO. In this approach, we let the CNN find the most informative bands from the existing ones using the FODPSObased feature selection. The overall accuracy of CNN+LR on validation samples was considered as fitness value. Based on obtained fitness values on validation samples, FODPSO iteratively tries to eliminate extra bands by considering many simultaneous parallel PSO algorithms, some specific punishment and reward rules, and using the concept of fractional calculus to model the dynamic of the swarm. Results indicate that the SICNN can significantly improve the CNN+LR in terms of classification accuracies and can classify hyperspectral data when there are only a limited number of training samples are available. In addition, in this paper, the concept of dither was proposed to efficiently regularize CNNs to improve the classification performance of the proposed method.

Both CNN and SICNN extracts spatial information using a fixed neighborhood system. In order to improve the classification accuracy, one can consider adaptive neighborhood systembased approaches such as morphological profile, attribute profiles, and segmentation approaches.

\section{ACKNOWLEDGMENT}

The authors would like to sincerely appreciate Prof. Paolo Gamba from the University of Pavia, Italy and Prof. David Landgrebe from Purdue University to provide Pavia University and Indian Pines data sets, respectively.

\section{REFERENCES}

[1] G. E. Hinton, S. Osindero, and Y. Teh, "A fast learning algorithm for deep belief nets," Neural Comp., vol. 18, no. 7, pp. 1527-1554, 2006.

[2] R. Salakhutdinov and G. E. Hinton, "Deep boltzmann machines," Int. Conf. Art. Intel. Stat., pp. 448-455, Clearwater Beach, Florida, 2009.

[3] Y. Bengio, P. Lamblin, D. Popovici, and H. Larochelle, "Greedy layer-wise training of deep networks," Neural Inf. Proc. Sys. 19, pp. 153-160, Cambridge, USA, 2007.

[4] Y. Chen, Z. Lin, X. Zhao, G. Wang, and Y. Gu, "Deep learning-based classification of hyperspectral data," IEEE
Jour. Sel. Top. App. Earth Obs. Remote Sens., vol. 7, no. 6, pp. 2094 - 2107, 2014.

[5] Y. Chen, X. Zhao, and X. Jia, "Spectra-spatial classification of hyperspectral data based on deep belief network," IEEE Jour. Sel. Top. App. Earth Obs. Remote Sens., vol. 8, no. 6, pp. 2381-2292, 2015.

[6] A. Krizhevsky, I. Sutskever, and G. E. Hinton, "Imagenet classification with deep convolutional neural networks," Proc. Adv. Neural Inf. Process, pp. 1097 -1105, 2012.

[7] J. A. Benediktsson and P. Ghamisi, Spectral-Spatial Classification of Hyperspectral Remote Sensing Images. Artech House Publishers, INC, Boston, USA, 2015.

[8] P. Ghamisi and J. A. Benediktsson, "Feature selection based on hybridization of genetic algorithm and particle swarm optimization," IEEE Geos. Remote Sens. Let., vol. 12, no. 2, pp. 309 - 313, 2015.

[9] Y. Bazi and F. Melgani, "Toward an optimal svm classification system for hyperspectral remote sensing images," IEEE Trans. Geos. Remote Sens., vol. 44, no. 11, pp. 3374-3385, 2006.

[10] P. Ghamisi, M. S. Couceiro, and J. A. Benediktsson, "A novel feature selection approach based on FODPSO and SVM," IEEE Trans. Geos. Remote Sens., vol. 53, no. 5, pp. 2935-2947, 2015.

[11] A. J. R. Simpson, "Dither is better than dropout for regularising deep neural networks," arXiv:1508.04826, 2015.

[12] A. Simpsons, "Over-sampling in a deep neural network," arxiv.org abs/1502.03648, 2015.

[13] P. Ghamisi, M. S. Couceiro, J. A. Benediktsson, and N. M. F. Ferreira, "An efficient method for segmentation of images based on fractional calculus and natural selection," Expert Syst. Appl., vol. 39, no. 16, pp. $12407-$ 12 417, 2012.

[14] P. Ghamisi, J. A. Benediktsson, G. Cavallaro, and A. Plaza, "Automatic framework for spectral-spatial classification based on supervised feature extraction and morphological attribute profiles," IEEE Jour. Sel. Top. App. Earth Obs. Remote Sens., vol. 7, no. 6, pp. $2147-$ 2160, 2014. 